\title{
Geothermal energy
}

\author{
A. Manzella \\ Institute of Geosciences and Earth Resources - Pisa, Italy
}

These lecture notes were originally published in EPJ Web of Conferences, 148 (2017) DOI: $10.1051 /$ epjconf/201714800012 as a contribution to the Proceedings of the 5th course of the MRS-EMRS "Materials for Energy and Sustainability" and 3rd course of the Joint EPS-SIF International School on Energy held in 2016. They are here reproduced as a good summary of the material presented in July 2017 during the course "Advances in Basic Energy Issues". 